\title{
Ständige Selbst- und Fremdbeobachtung
}

\author{
Ein E-Mail-Wechsel über Twitter
}

Dieses Gespräch hat zwei Ausgangspunkte: Die Studie Die soziale Logik des Likes (2018) von Johannes Paßmann und den Workshop „Schreibweisen der Gegenwart: Digitale Lektüren, digitale Texte“ des DFG-Projekts „Schreibweisen der Gegenwart. Zeitreflexion und literarische Verfahren nach der Digitalisierung“, der am 10. Dezember 2020 in digitaler Form an der Universität Greifswald stattgefunden hat. ${ }^{1}$ Es handelt sich um die schriftliche Reinszenierung und Fortsetzung eines Gesprächs, das Elias Kreuzmair dort mit Johannes Paßmann geführt hat. Im Zentrum steht die Frage, was Twitter aus Sicht der Medienwissenschaften einerseits und der Literaturwissenschaften andererseits ausmacht.

Deine Dissertationsschrift Die soziale Logik des Likes ist eine „Twitter-Ethnografie“. Wie kamst Du zum Thema Twitter? Und bist Du im akademischen Umfeld auch auf Widerstände gestoßen?

Ich habe 2010 eigentlich eine Dissertation zu einem ganz anderen Thema begonnen, im Graduiertenkolleg „Locating Media“. „Mediale Grenzen“ war der Arbeitstitel, ich habe mich damals mit der Frage befasst, welche Qualitäten nicht-digitaler Medien von Dauer bleiben. Meine Ausgangsbeobachtung war: Digitale Medien können alles emulieren, allerdings nicht die Begrenztheit der alten Medien. Ich war vorher Hilfskraft in einem Projekt von Jörg Döring und Tristan Thielmann zu Geomedien; dort war diese Frage ebenfalls virulent: Was hat die Papierkarte, was Google Maps nicht hat?

Vor diesem Hintergrund war Twitter besonders interessant, denn ich verfolgte eine Art Kompensationsthese: Weil digitale Medien sozusagen einen Grenzmangel haben, müssen sie und ihre Nutzung pragmatische Grenzen ziehen. Dass Twitter mit seiner harten 140-Zeichen-Grenze damals so ungeheuer erfolgreich war, hat mich deshalb interessiert: Offenbar, so dachte ich, ,braucht‘ digitale Kommunikation eine Logik der begrenzten Einheit, die alte Medien qua Materialität immer schon hatten. Das war aber gar nicht mal der Hauptgrund. Letztlich

1 Vgl. Johannes Paßmann: Die soziale Logik des Likes. Eine Twitter-Ethnografie. Frankfurt a. M./New York 2018.

Ә Open Access. (C) 2022 Elias Kreuzmair et al., publiziert von De Gruyter. (c) BY-NC-ND Dieses Werk ist lizenziert unter einer Creative Commons Namensnennung - Nicht-kommerziell - Keine Bearbeitung 4.0 International Lizenz. https://doi.org/10.1515/9783110758603-012 
habe ich damit begonnen, weil ein Freund schon ziemlich lang dabei war und öfters Ideen in Tweets ,umwandelte', das war spannend, da wollte ich dabei sein.

Twitter hat mich damit ziemlich schnell aufgesogen, diese ,Umwandlung“ von Beobachtungen in Tweets hat in dieser Zeit regelrecht Besitz von meinem Denken ergriffen. Ich bemerkte, wie ich für ein ganz anderes Beobachten sensibilisiert wurde - ein Vorgang, der mir aus der phänomenologischen Gestalttheorie bekannt vorkam. Ich hatte meine Diplomarbeit kurz vorher über den Begriff des Feldes bei Kurt Lewin, Wolfgang Köhler und Ernst Cassirer geschrieben. Ohne darauf jetzt zu weit eingehen zu wollen: Diese Frage neuer, alltäglich wirksamer Deutungsschemata, also neuer Hinsichten, unter denen Beobachtung organisiert wird, ist dort zentral. Lewin beschreibt etwa in „Kriegslandschaft“, einer phänomenologischen Studie von 1917, wie sich Raumwahrnehmung dadurch transformiert, dass man eine Landschaft statt in Friedenszeiten in Kriegszeiten durchschreitet. ${ }^{2}$ Das ist natürlich ein ganz anderer Fall, allerdings geht es bei beidem - der „Kriegslandschaft“ wie dem Twittern - um eine funktionalisierte Beobachtung von Umwelt: $X$ kann ich neuerdings für $Y$ brauchen. Mir fiel auf, wie ich durch Twitter die Nachrichten anders lese, die Menschen und ihr Tun an der Supermarktkasse anders beobachte, ihr Verhalten im Zug. Dabei kam mir dann stets der Gedanke: „Ist es nicht auch komisch, dass ...“. Da habe ich versucht, sie in Tweets ,umzuwandeln', und da merkt man schnell, was funktioniert und was nicht. Daran passt man die Beobachtung dann an, versucht, anders ,umzuwandeln', nimmt andere Aspekte an Alltagsbeobachtungen wahr.

Der Freund, der mich als Novize bei Twitter eingeführt hat, hatte mir nämlich einen ganz bestimmten Teil Twitters gezeigt, wie ich erst später bemerkte. Zentral für die Gruppe war etwas, was Jahre später zentral für ganz Twitter und viele andere Plattformen wurde: Die Beobachtung der Popularität der eigenen Texte durch Retweets, Favs, später Likes und so weiter. Twitter zeigte erhaltene Favs und Retweets damals noch nicht so an wie heute, der Drittanbieter Favstar.fm allerdings sehr wohl (und sogar in größerem Umfang - so hatte man eine persönliche Best of-Liste mit den populärsten Tweets, die einem je gelungen sind). Das hat einerseits zu einer ganz bestimmten Sensibilisierung der Beobachtung geführt, denn wenn man sieht, wie erfolgreich so ein Tweet werden kann, will man natürlich wissen, wie weit man es schaffen kann, warum es die anderen schaffen und so weiter. Man beobachtet die Welt daher immer mehr hinsichtlich der Frage, was sich in einen populären Tweet umwandeln lässt.

2 Kurt Lewin: „Kriegslandschaft“ [1917]. In: Feldtheorie. Werkausgabe. Hg. v. Carl Friedrich Graumann. Bd. 4. Bern/Stuttgart 1982, S. 315-325. 
Andererseits passiert bei diesen Popularitätsmarkern ja mehr als eine bloße Messung der Popularität eines Tweets. Man bekommt den Fav, Like und Retweet ja immer von jemand ganz bestimmtem und beobachtet dann natürlich auch diese $^{\star} n$ Andere ${ }^{\star} n$ auf ganz bestimmte Weise. Das kam mir aus der Ethnologie der Gabe sehr bekannt vor, mit der sich der Sprecher unseres Graduiertenkollegs Erhard Schüttpelz intensiv befasst hat. Mich hat das eigentlich nie so recht interessiert und auch Schüttpelz' medientheoretische Arbeiten schienen mir damals eher abwegig, aber nun merkte ich, wie viel man über Twitter lernen kann, wenn man Theorien der Gabe liest. ${ }^{3}$ Ich berichtete ihm daher von meinen Twitterbeobachtungen. Er sagte mir, diese Dissertation über mediale Grenzen, das wäre vielleicht nicht die beste Idee, viel interessanter wäre doch eine Ethnografie über das Twittern. Ich hatte ihm auch von Twitter-Treffen erzählt, wie die Online-Interaktion in die Krise kam, wenn man sich jenseits der Plattform begegnete, und da war er so entflammt, dass mir kaum noch eine Wahl blieb.

Dass ich auf Widerstände gestoßen bin, kann ich insofern wirklich nicht sagen. Ganz im Gegenteil, mein akademisches Umfeld wollte, dass ich genau diese Arbeit schreibe. Der größte Widerstand dabei kam eigentlich von mir selbst. Ich wollte ein großer Medientheoretiker werden und kein Twitter-Versteher.

Mir fällt auf, dass Du in Deiner Antwort immer wieder den Begriff ,umwandeln“ benutzt. Ich würde den Begriff gerne mit einem Satz in Verbindung bringen, den Du an anderer Stelle in einem gemeinsam mit Cornelius Schubert verfassten Aufsatz zur „Technographie als Methode der Social-Media-Forschung“ geschrieben hast: „Technik und Medien sind immer Medium und Resultat gesellschaftlicher Prozesse, d.h. sie konstituieren Gesellschaft und werden durch sie konstituiert. “4 Ist die ,Umwandlung', wie Du sie beschreibst, nicht auf Mikroebene das, was ihr hier auf der Makroebene beschreibt? Und was hieß das wiederum für das methodische Vorgehen der „Twitter-Ethnografie“?

Mit der ,Umwandlung، meine ich zunächst, dass das Twittern einem eine Entwicklung poetischer Skills nahelegt, sei es durch Verdichtung, Zuspitzung, Verkettung einzelner ,Bullet Points‘ oder eben den Verzicht darauf, durch Pointen, den Entwurf einer bestimmten Persona und so weiter. Jeder dieser Texte ist öffentlich, begrenzt, und in jeden dieser Texte sind Bewertungsparatexte eingebaut. Egal, was man von Likes hält, jeder Tweet hat einen Like-Counter. Man kann sich dazu entscheiden, sich davon nicht beeindrucken zu lassen, aber selbst dann positioniert man sich schon zu einer Bewertung. Man darf diese Praktiken natürlich nicht allein auf deren zählbare Bewertung reduzieren, auch

3 Siehe für einen instruktiven Überblick Iris Därmann: Theorien der Gabe. Hamburg 2010.

4 Johannes Paßmann/Cornelius Schubert: „Technografie als Methode der Social-Media-Forschung. “ [Preprint] In: Netzwerk Diskurse Digital (Hg.): Handbuch Diskurse Digital. Berlin/Boston 2021, i.Beg. DOI: 10.13140/RG.2.2.22738.50883, S. 8. 
all die anderen Reaktionen, Nicht-Reaktionen und Präsenzen motivieren dazu, eigene Techniken des Schreibens zu entwickeln.

Was Du beschreibst, wäre schon ein Element dessen, was Du als „soziale Logik des Likes“ bezeichnet hast, wenn ich es richtig sehe. Wie schließen die vorhin schon angesprochenen Überlegungen zur Theorie der Gabe an diese Analyse der „poetischen Skills“ der Twitternden an?

Ganz genau, bei der „sozialen Logik“ geht es nicht darum, dass die Plattform-Einheiten bestimmte, determinierbare Effekte haben, sondern dass sie Praktiken der wechselseitigen Beobachtung initiieren und verstetigen können. Der erhaltene Like, der erhaltene Follower regt zu der Frage an, welche Bedeutung diese Gabe hat. Ist dies eine Auszeichnung für einen hochwertigen Text, den ich geschrieben habe? Ist es ein wertloses, inflationär vergebenes Werbegeschenk, das mich nur dazu bringen soll, mich verpflichtet zu fühlen und mich zu revanchieren? Alle Social-Media-Plattformen regen diesen Kreislauf aus Gabe und Gegengabe an; immer gibt es Texte, Bilder, Klänge, die mit Plattform-Einheiten bewertet werden, die selbst wiederum konkreten Accounts mit Kopf und Zahl zugeordnet sind. Das heißt natürlich nicht, dass daraus ein reziprokes Verhältnis erwächst. Die quantitativen Daten zeigen meist gar eher das Gegenteil: Es gibt meist „Power-Law-Distributions“, ${ }^{5}$ bei denen ganz wenige ganz viel erhalten und ganz viele ganz wenig. Aber auch das ist ja typisch für viele Gabentausch-Beziehungen, dass sie asymmetrisch bleiben.

Es gibt also eine ständige Selbst- und Fremdbeobachtung der eigenen Texte, dies nicht nur, weil sie ständig bewertet werden und ständig von Accounts mit unterschiedlicher Bedeutung bewertet werden (es gibt etwa Accounts, deren Anerkennung wir sehr wertschätzen, weil wir die Betreiber`innen selbst sehr anerkennen), ${ }^{6}$ sodass wir auch ständig beobachten können, wer genau uns beobachtet. Vielmehr können wir auch mit jedem Like, jedem Retweet und so weiter unsere eigenen Texte immer wieder neu und immer wieder anders lesen. Denn im Notifications-Tab wird uns der Post jedes Mal neu vorgelegt, sodass wir ihn im Lichte der Bewertung neu wahrnehmen können.

Das hat Folgen für die Texte, da ja die Plattformeinheiten wie Retweets und Likes eben stets für einzelne Texte vergeben werden. Man kann sich etwa ermutigt fühlen, so zu schreiben, dass ein Tweet viral geht oder so, dass man nur in

5 Albert-László Barabási/Eric Bonabeau: „Scale-Free Networks“. In: Scientific American 288/1 (2003), S. 60-69. Auch bekannt als,Long Tail': Chris Anderson: The Long Tail - der lange Schwanz. Nischenprodukte statt Massenmarkt - Das Geschäft der Zukunft. München 2007.

6 Siehe hierzu das Kapitel „Die Autoritätsbindung“ in Heinrich Popitz: Phänomene der Macht [1986], 2., st. erw. Aufl. Tübingen 2009, S. 104-131. 
einem bestimmten Milieu Anklang findet. Man kann sich für ausbleibende Reaktion schämen oder so schreiben, dass die Texte nicht auf Popularität angelegt scheinen. Wie auch immer Texte auf diese digitalen Paratexte reagieren, die Möglichkeiten sind vielfältig; bloß eine Möglichkeit gibt es nicht: Sie unwirksam zu machen.

Wir forschen im Projekt „Schreibweisen der Gegenwart“ insbesondere zu Zeitkonzepten in literarischen und zeitdiagnostischen Texten, die in Bezug zur Digitalisierung stehen. Deswegen ist eine Passage aus der Einleitung von Die soziale Logik des Likes auf unser besonderes Interesse gestoßen. In Bezug auf Soziale Medien schreibst Du: „Jede und jeder dort hat ein großes soziales Netz mit schwachen Verknüpfungen, die man aus guten Gründen etwas verstetigen kann - allein schon, um sich zu Parties einzuladen, für Lern- oder Sexualkontakte und all die anderen zentralen Praktiken des Studierens. Sozialpsychologisch besonders wichtig ist dafür die Zeitlichkeit der Plattformen." ${ }^{7}$ Warum ist die Zeitlichkeit besonders wichtig? Und wie genau würdest Du sie beschreiben?

An der Stelle des Buchs geht es um die Frage, warum die Social-Media-Plattformen erst an den Universitäten groß wurden; die ersten wirklich populären Plattformen in Europa und den USA waren ja StudiVZ und Facebook. Meine These dort ist, dass in den Universitätskulturen schon lange Praktiken und Technologien etabliert waren, die für diese Plattformen sehr gut anschlussfähig waren. Ein Großteil der Studierenden, und zwar gerade die, die das Studium beginnen, ist in einer Liminalitätsphase. Die Stabilisierung all der vielen neuen und oft nur flüchtigen Beziehungen ist da ebenso wichtig wie die Selbstbeobachtung im Lichte der Beobachtung der Anderen. Diese Form der Beobachtung ermöglichen die Plattformen auf besondere Weise, indem sie Interaktion extrem verlangsamen. Die Erwiderung eines Kontaktangebots kann ja durchaus Tage dauern.

Mit dieser Langsamkeit der Plattformen gehen nicht nur Subjektivationspraktiken einher, sondern gleichzeitig immer auch solche der Objektivation. ${ }^{8}$ Die Langsamkeit der Beobachtung bezieht sich ja etwa auch auf die Beobachtung und Herstellung von Texten, Bildern und Klängen. Wenn uns etwa mit jedem Like derselbe Tweet noch einmal vorgelegt wird, kehrt man ja immer wieder zu denselben zwei, drei Sätzen zurück. Und wenn dasselbe eine Bild Bernie Sanders' bei der US-Präsidentschaftsinauguration zigtausendfach ein- und ummontiert wird, ist das zwar einerseits eine unheimliche Flut unterschiedlicher

7 Paßmann: Die soziale Logik des Likes, S. 13.

8 Siehe auch Johannes Paßmann/Cornelius Schubert: „Objektivation online. Subjekte und Objekte sozialer Medien“. In: MedienJournal. Zeitschrift für Medien- und Kommunikationsforschung. Special Issue: Medien als Dinge denken. Zur Materialität des Digitalen Hg. v. Anja Peltzer u. a., 2020/4 (i. E. 2021), DOI: 10.13140/RG.2.2.11361.74085. 
Bilder in enger Taktung, aber eben auch eine enorme Beschäftigung mit dieser einen Person in dieser einen Pose, mit der sich jede ${ }^{\star}$, die oder der Sanders in ein Bild montiert, intensiv befasst: Wo muss ich den freigestellten Bernie genau positionieren? Passt dieser eine Hintergrund perfekt oder sollte es lieber ein anderer sein? Was ist dieser Bernie eigentlich für ein straighter Typ, da mit diesen Strickfäustlingen zu sitzen, während selbst die Dichterin Prada trägt? ${ }^{9}$

Und wenn wir drei Bernie-Bilder gelikt haben, wissen wir auch schon ziemlich genau, wie seine Handschuhe aussehen oder seine FFP1-Maske in dieser Sekunde saß. Das hat auch mit der Art und Weise zu tun, wie uns diese Bilder, Texte und Klänge immer wieder vorgelegt werden. Wir können Zoomen, wir können das Tempo manipulieren, wir können Elemente de- und rekomponieren und wir werden gebeten uns dazu zu positionieren, per Like, Kommentar oder Weiterscrollen. Während mir also ziemlich offenkundig scheint, dass die Plattformen Medien der Beschleunigung sind, so scheint mir der erwähnenswerte Aspekt vor allem ihre gleichzeitige Verlangsamung zu sein.

Diese Gleichzeitigkeit der verschiedenen Geschwindigkeiten steckt meines Erachtens im Begriff der ,ballistischen Ästhetik‘, über die Maren Jäger auf unserer gemeinsamen Tagung gesprochen hat. ${ }^{10}$ Ballistik ist die Lehre der geworfenen Körper und hat insofern eine natürliche Nähe zur Geschwindigkeit. Man kann Ballistik aus meiner Sicht allerdings auch als eine Technik der extrem verlangsamten Beobachtung dieses Geworfenen verstehen. Ballistik schießt und wirft ja nicht nur, sie zeichnet vor allem die Bahnen ihrer geworfenen und geschossenen Objekte minutiös nach und fragt, wieso die Verlaufsbahn gerade diese eine und nicht eine andere Form hat. Genau das tun wir jeden Tag auf den Plattformen. Im Notifications-Tab, in Buzzfeed-Artikeln, im Entrollen von Threads oder in der Entscheidung, uns nach diesem Diskussionsverlauf doch lieber nicht auch noch einzumischen. Oder indem wir uns den dreitägigen Hype um die Bernie-Memes nacherzählen. Immer zeichnen wir die Trajektorie dieser Texte, Bilder und Klänge nach. Diese Verlaufsbahnen beschreib- und be-

9 Vgl. O.A.: „Bernie Sanders Wearing Mittens Sitting in a Chair“. In: Know Your Meme. https://knowyourmeme.com/memes/bernie-sanders-wearing-mittens-sitting-in-a-chair, Februar 2021 [zuletzt eingesehen am 7.6.2021].

10 Es geht um den in der Einleitung angesprochenen Workshop „Schreibweisen der Gegenwart: Digitale Lektüren, digitale Texte“ am 10. Dezember 2020. Einen Überblick über die dortige Diskussion geben Elias Kreuzmair/Magdalena Pflock: „Über Twitterliteraturwissenschaft“. In: Schreibweisen-Blog. https://germanistik.uni-greifswald.de/institut/arbeitsbereiche/ neuere-deutsche-literatur/dfg-projekt-schreibweisen-der-gegenwart/schreibweisen-blog/n/ ueber-twitterliteraturwissenschaft-81958/, 17.12.2020 [zuletzt eingesehen am 7.6.2021]. 
obachtbar zu machen, war vielleicht schon immer die entscheidende Stärke der Social-Media-Plattformen.

\begin{abstract}
Bei Maren Jäger, die den Begriff der ,ballistischen Ästhetik‘ von Joseph Vogl entleiht, ging es mit Blick auf kurze Texte seit der Antike um Text-Geschosse, die ein*e Sender*in in Richtung eines*einer Empfänger*in abschießt. Auf Twitter wäre damit wohl die Rhetorik von Donald Trump angesprochen, der mit seinen Tweets immer wieder politische Gegner*innen angegriffen hat. Insofern finde ich an deiner Wendung des Ballistik-Begriffes interessant, dass du ihn als ein Verfahren der Beobachtung einführst. Man müsste dann aber sagen, dass die Verlaufsbahnen, die man beobachten kann, sich in komplexeren Zirkulationsmustern bewegen als nur von einer Person oder Gruppe zu einer anderen Person oder Gruppe. Die Verlaufsbahnen entstehen doch eher in einem Prozess, in der viele durch die Aktionen beteiligt sind, die Du beschreibst. Zudem stellt sich mit der Ballistik als Technik der Beobachtung von Verlaufsbahnen die Frage, wie man diese neuen Plattformen im Allgemeinen und Twitter im Speziellen überhaupt adäquat beobachten kann. Was waren methodische Leitideen für deine Studie? Was zeichnet die Ethnografie als Verfahren der Medienwissenschaft aus? Lassen sie sich in Bezug zu einer Ballistik stellen, wie Du sie jetzt beschreibst?
\end{abstract}

Genau, man kann das Ballistische als Schreib- und als Beobachtungstechnik verstehen, und vor allem darf man die Metapher nicht überladen; allein schon, weil die Kraft der Bewegung beim Tweet nicht nur vom Text und seinem Ursprung ausgeht, sondern auch von dem Netzwerk, das ihn zirkuliert und insofern mehr ist, als die Wind- und Wasserverhältnisse der Ballistik. Das heißt, so sehr Ballistik für die Verfahren der Beobachtung sensibilisiert, so sehr rückt sie Sender`in und Empfänger`in über Gebühr in den Mittelpunkt - und degradiert das restliche Netzwerk zu passiven Objekten.

In meiner Twitter-Ethnografie habe ich ja vor allem auch zu zeigen versucht, dass es nicht nur darauf ankommt, die richtigen Texte über die Plattform zu schießen, sondern dass es spezifischer sozialer Skills bedarf, durch die man ein Netzwerk aktiv aufbaut, eine Community von Followern und Gefolgten, mit denen man regelmäßig in Austausch tritt. Teils funktioniert das per Gabe und Gegengabe von Likes und anderen Plattform-Einheiten, mit denen man sein Netzwerk täglich (re-)produziert, pflegt und erweitert, teils durch andere Praktiken. In dem Feld, das ich untersucht habe (die ,Favstar-Sphäre' zwischen 2011 und 2015) gab es auch viele persönliche Treffen, die diese wechselseitigen Verpflichtungen angebahnt, verstärkt oder teils auch zerstört haben. Dabei fiel mir dann aber auf, dass ich zwar als Teilnehmer sehen konnte, wie stark wechselseitige Verhältnisse aufgebaut wurden, dass darüber aber niemand sprach; und wenn doch, dann wurde in diesem Reden über die Praktiken der Reziprozität meist eher verdeckt, als das erläutert wurde, wie sie funktionieren. Es war nicht nur so, dass die User`innen nicht erklären wollten, wie es funktioniert, sie suchten sogar Ausreden, wieso es anders funktioniert, als es scheint: Die Favs, Retweets und Follower hätten alle keine Bedeutung für sie, es kränke sie nicht, 
wenn jemand ihre Tweets plagiiere und damit selbst Anerkennung einheimse, die ihnen gebührt und natürlich erwarte man nicht, dass andere sich revanchierten, wenn man etwas für die Zirkulation ihrer Tweets tat.

Das ist aber doch erst einmal ein Problem für Forscher*innen, wenn die User*innen nicht erklären wollen, wie es funktioniert. Beziehungsweise: Man macht gewissermaßen erst einmal eine Beobachtung zweiter Ordnung, merkt aber, dass man an die eigentliche Praxis nicht herankommt. Auf welche Weise werden diese Praktiken, abgesehen von der Beobachtung des eigenen Vorgehens, sichtbar? Gibt es bestimmte Situationen, in denen sie doch thematisiert werden?

In eigentlich allen Kulturtheorien anthropologisch stabiler sozialer Medien wie verschiedenen Formen des Geldes und der Gabe gibt es genau dieses Problem, das in diversen Varianten der Regel aufscheint, diese Medien selbst nicht zu thematisieren. Insofern etwa die Gabe häufig Anerkennung erzeugt, kann es leicht zum Problem werden, ihre Bedeutung und Genese explizit zu machen, vornehmlich aus zwei Gründen. Zum einen kann die Explikation Fragen aufwerfen, die den symbolischen Akt der Anerkennung zunichte machen: Ein Geschenk, nach dem gefragt wird, wird nicht mehr aus freien Stücken übergeben. Ein Geschenk, dessen Funktion und Ziel erklärt wird, kann leicht mit imaginierten Zielen konfligieren. Und ein empfangenes Geschenk, dessen Funktion und Ziel hinterfragt werden, kann die Nehmerin sehr leicht als abhängig von diesem Geschenk, als heteronom erscheinen lassen. Während Heteronomie gegenüber unseren Formen des Geldes allerdings relativ weit akzeptiert ist, gerät man mit dem Eingeständnis einer Heteronomie gegenüber den empfangenden Followern und Likes leicht ins Lächerliche.

Der zweite Grund ist, dass Soziale Medien ihre Kraft oft aus einer Vagheit beziehen, einer Vagheit, die nicht aufgelöst wird. Bei Karl H. Hörning habe ich für solche Phänomene den Begriff der ,produktiven Unbestimmtheit' gefunden, ${ }^{11}$ das fand ich für die Plattformeinheiten ebenso passend wie für viele Emojis und Sticker in Chatprogrammen wie WhatsApp, Telegram oder Signal, und nicht zuletzt halte ich dies für eine allgemeine Stärke vieler Sozialer Medien: Ihre Produktivität liegt häufig darin, bestimmte Information gerade nicht zu übertragen; dies Mal, weil sie ,vorhandene‘ aber den sozialen Prozess störende Information ,schlucken', und mal, weil sie Konkretion schaffen, wo ohne sie keine vorläge. Denken wir neben den Likes und Favs etwa an all die Tierchen- und Gemüse-

11 Karl H. Hörning: „Lob der Praxis. Praktisches Wissen im Spannungsfeld zwischen technischen und sozialen Uneindeutigkeiten“. In: Gerhard Gamm/Andreas Hetzel (Hg.): Unbestimmtheitssignaturen der Technik. Eine neue Deutung der technisierten Welt. Bielefeld 2005, S. 297-310, hier S. 308. 
Emojis, an all die Sticker-Varianten, die selbst wiederum an die Emojis rückgekoppelt sind. Dort geht es ja gerade nicht um präzise Informationsübertragung und auch nicht unbedingt um eine Komplexitätsreduktion, sondern häufig um ebendiese produktive Unbestimmtheit. Die kann freilich im Gebrauch in bestimmten Kontexten enorme Bedeutungsstabilität erlangen, wie etwa im Fall der Aubergine oder des Pfirsichs, dies ist aber nur der Fall, weil dies eine situative Aneignung des prinzipiell Unbestimmten ist, dem seine symbolische Unbestimmtheit immer noch anhaftet. Wenn es etwa ein Emoji eines erigierten Penis gäbe, würden viele vermutlich doch lieber bei der Aubergine bleiben.

Wenn es also ein zentrales Charakteristikum Sozialer Medien ist, ihre Funktion in der Latenz zu belassen, resultiert daraus das Problem, dass sich zwar ihre Praxis, aber nicht ihre Praktiken beobachten lassen: Ich sehe zwar, was geschieht, habe aber keinen Zugriff auf das, was die Regelmäßigkeit dieses Geschehens ausmacht. Das Sprechen über diese Praktiken ist so eine ganz eigene Praktik mit ganz eigener Funktion: Ebendiese Latenz zu erhalten.

Dies verweist auf ein Grundproblem, das Medienwissenschaft und Praxeologie gleichermaßen schon immer hatten, das Medium entzieht sich im Gebrauch, wie wir schon bei Fritz Heider lesen können ${ }^{12}$ und bereits für Talcott Parsons ist es zentral, dass die reproduzierten Wertmuster in der Latenz bleiben - das L seines AGIL-Schemas steht ja für die „Latency“. ${ }^{13}$ Die Lösung für dieses Problems ist meist recht ähnlich: Bei Fritz Heider macht die Störung das Medium beobachtbar, und Parsons' Schüler Harold Garfinkel ist auch dadurch bekannt geworden, dass er die latenten Muster der Praxis durch die , breaching experiments ${ }^{\text {` }}$ die Krisenexperimente - aufschließbar machte. ${ }^{14}$

Solche absichtlichen Disruptionen sind heute aus forschungsethischen Gründen nicht mehr üblich, sie sind aber für den Fall der Social-Media-Ethnografie aus meiner Sicht auch nicht nötig. Denn solche Disruptionen ereignen sich so oder so ständig. Meine Forschungsmaxime war deshalb, mich für solche Momente der Störung zu sensibilisieren und hinzuschauen, wenn sie geschehen. Es gibt ja eine Reihe ethnografischer Maximen, die je Abwandlungen von Bronisław Malinowskis „Follow the Natives“ darstellen, wie Bruno Latours „Follow the Actors“ oder die ganze Reihe von Folgeimperativen, die George E. Marcus in Ethnography through Thick \& Thin genannt hat: „Follow the People, Follow the Thing, Follow the Metaphor, Follow the Plot, Story, or Allegory, Follow the Life or Biogra-

12 Vgl. Fritz Heider: Ding und Medium. Berlin 2005 [1926].

13 Vgl. Talcott Parsons: The Social System. London 1951.

14 Harold Garfinkel: „Studien zu den Routinegrundlagen von Alltagstätigkeiten“ [1967]. In: Ders.: Studien zur Ethnomethodologie. Übers. v. Brigitte Luchesi, hg. v. Erhard Schüttpelz u. a.. Frankfurt a.M./New York 2020, S. 77-126. 
phy“ und so weiter. ${ }^{15}$ Für die Social-Media-Ethnografie habe ich mir in diesem Sinne die Maxime „Follow the Disruptions“ vorgenommen und drei Typen von Disruptionen unterschieden: (1) Technische Störungen, wie Defekte oder Updates, (2) Störungen sozialer Regeln, die etwa durch Tabubrüche entstehen oder teils in sogenannten Shitstorms beobachtbar werden und (3) Störung durch technischen Entzug, wie sie etwa dann eintritt, wenn man User*innen, die man teils seit Jahren über Twitter kennt, zum ersten Mal persönlich trifft.

Wenn man die Liste von George E. Marcus liest, kommen dort einige Begriffe vor, die man durchaus mit literarischen Texten assoziieren könnte. Zum Beispiel ,story', ,plot', ,metaphor oder ,allegory'. Ich habe mich in diesem Zusammenhang gefragt, ob man nicht auch literarische Texte als eine Form der Störung verstehen könnte, in der das Medium reflektiert wird. Ein Beispiel: Der Schriftsteller Saša Stanišić schreibt am 12. Juli 2020 auf Twitter: „Ahnenpaß hamburg wo fälschen“ und ergänzt diesen Tweet kurz darauf mit „sorry falsceh [sic!] suchmaske ${ }^{\text {"16 }}$. Stanišić schließt damit thematisch lose an seinen im Jahr 2019 veröffentlichten Roman Herkunft an und greift zugleich die Debatte über von Seiten der AfD mit mehr oder weniger deutlichem Bezug zu entsprechenden in nationalsozialistischer Terminologie formulierten Fantasien über eine vermeintliche „deutsche Leitkultur“ auf. ${ }^{17}$ Die kurze Erzählung in zwei Tweets - ein Mann aus Hamburg will einen „Ahnenpass“ fälschen und „sucht“ versehentlich bei Twitter statt mit einer Suchmaschine - lässt doch einige Rückschlüsse auf das Medium zu, in dem sie erzählt wird. Sie inszeniert verschiedene Schreibkonventionen in unterschiedlichen Medien und stell diese gegeneinander: Im ersten Tweet wird gezeigt, wie Suchmaschinen befragt werden, im zweiten wird der Ton auf Twitter als umgangssprachlich markiert, wobei Flüchtigkeitsfehler die Regel sind und nicht sanktioniert werden. Die mangelnde Medienkompetenz des Protagonisten dieser Kürzestgeschichte akzentuiert zugleich die Medienkompetenz des Autors Saša Stanišić, der eben weiß, wo man sich wie zu artikulieren hat und damit umzugehen versteht. In diesem Sinn verweist die Veröffentlichung der Kürzestgeschichte auch darauf, dass auf Twitter intimere Kommunikation mit Autor*innen möglich ist, dass man sich aber nie sicher sein kann, wie authentisch deren Selbstinszenierung ist. Insofern dient diese Erzählung auch dazu, die von dir beschriebene produktive Unbestimmtheit zu erhalten, würde ich sagen. Zuletzt könnte man das Thema der Kürzestgeschichte als Beleg dafür heranziehen, dass Twitter ein Ort der Kommunikation über politische Themen ist, die auch in dieser Erzählung aufgegriffen werden. Oder würde das der Medienwissenschaftler alles ganz anders sehen?

Das ist ein sehr interessantes Beispiel, wie die Imitation als bekannt vorausgesetzter digitaler Schreibpraktiken literarisch produktiv gemacht wird. Dabei inszeniert er aber noch eine andere Schreibkonvention: Die alte Rechtschreibung

15 George E. Marcus: Ethnography through Thick \& Thin. Princeton, NJ 1998, S. 89 ff.

16 Saša Stanišić [@sasa_s]: „Ahnenpaß hamburg wo fälschen“ [Tweet]. https://twitter.com/ sasa_s/status/1282278175788150786, 12.7.2020 [zuletzt eingesehen am 7.6.2021].

17 Etwa hier: Carsten Korfmacher: „AfD-Rechtsprofessor fordert arische Leitkultur“. In: Nordkurier. https://www.nordkurier.de/mecklenburg-vorpommern/afd-rechtsprofessor-fordert-ari sche-leitkultur-2527728904.html, 2017 [zuletzt eingesehen am 7.6.2021]. 
(„Ahnenpaß“) lässt sich ja als Hyperkorrektur lesen und insofern als Signal, dass es sich bei dem Protagonisten um jemanden handelt, der seine eigene ,nicht-arische“ Herkunft verbergen will: Warum auch immer der ,Migrant‘ sich die Rechtschreibregeln des alten Deutschlands angeeignet hat, er schreibt dadurch sozusagen ,deutscher' als die meisten ,Deutschen“ - ist es laut seiner familiären Herkunft allerdings nicht. Mit der scheinbaren Medienverwechslung Twitter/Suchmaschine wird die Panik des verunsicherten Hyperangepassten vermittelt, dessen Lebenslüge aufzufliegen droht.

In diese Deutung lassen sich auch die Flüchtigkeitsfehler einreihen: Er will das Versehen schnell korrigieren, wobei weitere Fehler passieren - nicht nur der Buchstabendreher und die „suchmaske“ (statt „Fenster“ oder „Eingabefeld“), sondern überhaupt, dass er wie in einem Chat die vorherige Nachricht korrigieren zu wollen scheint (statt den Tweet zu löschen).

Dies ist ja eine gängige Psychologisierung vieler Rechter: Ihr Rassismus ist demnach auch die Hyperkorrektur des ${ }^{\star}$ derjenigen, der ${ }^{\star}$ die sich seiner ${ }^{\star}$ ihrer eigenen Rolle unsicher ist und daher die Traditionen der Gesellschaft, deren Werte er^sie zu verteidigen glaubt, stärker vertritt als jene, deren Zugehörigkeit unzweifelhaft ist: Er schreibt so, wie es im alten Deutschland Regel war (heute aber nicht mehr). Ich interpretiere den Tweet deshalb als Psychogramm eines verunsicherten Rechten, der einen ungelösten inneren Zugehörigkeitskonflikt in politisierte Aggression überführt. Dies gelingt Stanišić, indem er dessen Ängste in seinem situativen Mediengebrauch sichtbar macht: dem panischen Googeln.

Man könnte nun nach weiteren Tweets suchen, die panisches Googeln inszenieren und würde dann vermutlich weitere mehr oder weniger gelungene Fälle finden die zeigen, dass Stanišić damit an einer etablierten Twitterpraktik teilnimmt; panisches Googeln von Krankheiten, Fristen, was auch immer, womit stets ein ${ }^{\star}$ e sich unbeobachtet glaubende ${ }^{\star}$ r Protagonist*in einen unbeabsichtigten Einblick in seine ${ }^{\star}$ ihre psychische Lage gibt. Eventuell finden sich noch besser gelungene Fälle, die sich etwa dadurch auszeichnen könnten, dass die panischen Vertipper solche sind, die einem tatsächlich häufig begegnen. „falsceh“ scheint mir zum Beispiel kein allzu häufiger Vertipper von „falsche“ zu sein - „flasche“ kommt möglicherweise häufiger vor.

Jedenfalls erweist sich der Autor selbst als registerkompetent: Er hat digitales Schreiben verstanden, sowohl das panische Googeln wie auch die Twitterpraktik, panisches Googeln per vermeintlicher Eingabefeld-Verwechslung zu inszenieren; er kann bei den kuranten Social-Media-Praktiken mindestens ebenso sehr mitspielen, wie im Literaturbetrieb. Statt also zu versuchen, Twitterpraktiken in Buchschreibepraktiken zu überführen, tut er beides an seinem jeweiligen Ort. Von Versuchen, das Twittern in Buchform zu überführen gibt es ja viele Beispiele. 
Ein ästhetisch überzeugendes ist mir nicht bekannt, und das liegt aus meiner Sicht daran, dass sich Medienpraktiken nicht verlustfrei verpflanzen lassen. Dies verstanden zu haben, scheint mir Stanišićs Registerkompetenz mit auszumachen, aber eben auch, sich auf Twitter zu bedienen, sich irritieren und inspirieren zu lassen - was ja viele Autor^innen tun, wie etwa zuletzt Amanda Gorman, die beschrieb, wie sie durch die Tweets zur Kapitolstürmung ein Verständnis für die Situation bekommen habe, die sie dann poetisch verarbeitet hat.

Interessant, dass du die Kürzestgeschichte ganz anders liest. Dein Protagonist ist ein Rechter, während in meiner Lesart eine autofiktionale Figur auftritt, die gerade aus Furcht vor Rechten agiert. Meine Lesart ist dadurch eng an die Kopplung der Tweets an den Account des Autors geknüpft. Das könnte auf Twitter doch zu Problemen führen, wenn sich andere diese Kürzestgeschichte aneignen: Du hast in deiner Studie auch über die Geschichte des Retweets geschrieben. Wirkt sich diese Praxis nicht auch auf das Verständnis von Autorschaft aus? Was kann jemand damit ausdrücken, dass er oder sie Stanišićs Kürzestgeschichte retweetet? Käme man da schon in die Nähe von kollektiven Praktiken der Autorschaft? Oder ist der Retweet eher eine erweiterte Form des Likes?

Möglicherweise liege ich mit dieser Interpretation daneben, denn auch Deine Auslegung scheint mir plausibel: Die Hyperkorrektur „Ahnenpaß“ würde der Protagonist demnach aus Angst vor den Rassisten vornehmen: Er schreibt sozusagen ,deutscher als die Deutschen', um nicht aufzufallen. Aber auch unabhängig davon würde ich den Tweet dem Genre des gekonnten Nachäffens zuordnen - man imitiert einen Typus des Sprechens oder auch einen Typus einer Person, dessen Widersprüchlichkeit man in einer Pointe entlarvt. Accounts wie @1A_Entrepreneur, @andykassier oder @DaxWerner sind so etwa um 2017 damit bekannt geworden, Startup- und andere Formen des mehr oder weniger gut getarnten Macho-Unternehmersprechs nachzuäffen. Wenn so ein Verfahren nicht nur als Text populär wird, sondern als Praktik, weil sie „mitspielfähig“ wird, wie dies Thomas Alkemeyer und Nikolaus Buschmann nennen, ${ }^{18}$ strahlt sie aus. Andere Accounts eignen sich solche Schreibpraktiken an, formen daraus ihren eigenen Stil, imitieren ihn in den Replies und signalisieren so, dass sie verstanden haben. Dabei spielen Buchautor^innen häufig gern mit, nicht nur Saša Stanišić, auch Jasmin Schreiber zum Beispiel war bereits früh bei diesen Praktiken dabei und hat sogar ihren Twitternamen an einen Slang angepasst, der sich in diesem Milieu entwickelt hat.

18 Thomas Alkemeyer/Nikolaus Buschmann: „Praktiken der Subjektivierung - Subjektivierung als Praxis“. In: Hilmar Schäfer (Hg.): Praxistheorie. Ein soziologisches Forschungsprogramm. Bielefeld 2016, S. 115-136. 
Es handelt sich insofern schon um ein kollektives Tun, um eine Praktik im engsten Sinne: Eine Operationskette wird nicht nur wiederholt, sie wird jedes Mal anders wiederholt, in neue Kontexte eingepasst, kreativ erweitert oder oft auch schlecht imitiert. Gleichzeitig wird diese Ausführung der Praktik ständig bewertet; es gibt Maßstäbe, die gute Versionen der Schreibpraktiken von schlechten unterscheidet. Es bilden sich emische High-Low-Axiologien heraus, die zwar nicht mehr so viel mit der Unterscheidung hoher und niederer Literatur zu tun haben, aber dennoch von den Teilnehmenden nach ästhetischen Regeln als hoch- oder niederwertig unterscheidbar werden. Das macht die Freude und den Spaß am sprachlich ambitionierten Twittern aus, man findet die schönen Texte und vergemeinschaftet sich mit ihnen; mal - und das ist riskant - durch schreibendes Mitspielen, mal und das ist relativ voraussetzungslos - durch liken oder retweeten. Je mehr man mitspielen will, umso mehr muss man sich auf Augenhöhe begeben und einen Text produzieren; einen anspruchsvollen Paratext, der die Ästhetik des Haupttextes spiegelt. Die Teilnahme an der Praktik per Like oder Retweet hingegen ist ziemlich vage, es lässt sich meist nicht feststellen, wie weit das Verständnis der Schreibpraktik geht.

Auch beim niedrigen Mitspielniveau per Plattform-Einheiten wie Likes und Retweets handelt es sich natürlich um einen Paratext, und einen sehr wichtigen sogar. Denn die Einheiten und ihre Counter stellen die Popularität des Textes fest. Das hat dann wieder Auswirkungen auf die Bedeutung der Haupttexte; wenn man sieht, wie populär @DaxWerners Tweets werden, liest man ein zweites Mal, versucht zu verstehen, was daran so beachtenswert sein soll und entwickelt eine Sensibilität für diese spezifische Ästhetik. Indem man mit Plattform-Einheiten also solche niedrigschwelligen, Popularität indizierenden Paratexte produziert, nimmt man in der Summe doch ganz erheblich an der Bedeutungsproduktion teil, indem man mitentscheidet, welche Texte einer genaueren Beobachtung unterzogen werden.

Von kollektiver Autorschaft würde ich dennoch nicht sprechen wollen, denn die Unhaltbarkeit scharfer Grenzen bedeutet ja nicht die Abwesenheit von Unterscheidbarkeit. Die einzelne Handlung - man tippt einen Text in ein Eingabefeld - wird von einzelnen Personen vollzogen. Sie nimmt aber - wie jede Handlung - zugleich an Praktiken teil. Und sie nimmt auch häufiger und intensiver an Praktiken teil - dies auch mit mehr verschiedenen Teilnehmenden als wir es aus der Literaturgeschichte kennen. Diese mitspielfähige und mitspielende Form der Autorschaft ist insofern kollektiver als früher und dennoch nicht schlechterdings kollektiv. Vielleicht sollten wir deshalb statt von kollektiver Autorschaft von kollektiverer Autorschaft sprechen. Andererseits ist sie aber auch weniger kollektiv, weil Plattformen stets eine strikte Kopplung zwischen Account, Post und Plattformeinheiten herstellen. Jeder Text ist Text eines ein- 
zelnen Accounts, jeder Like ist der Like eines einzelnen Tweets, der selbst wiederum einem einzelnen Account zugeordnet ist. Tweets in Co-Autorschaft sind nicht vorgesehen, Likes auch nicht. Wie bei der klassischen Münze ist jeder Einheit immer genau ein Kopf zugeordnet, der für sie verantwortlich zeichnet.

Ich finde den Gedanken, von „kollektiverer“ Autorschaft zu sprechen sehr spannend. Ist es nicht ähnlich wie bei den sozialen Beziehungen, die durch die Plattform sichtbarer werden? Also: Werden hier nicht Prozesse der Bedeutungs- und Textproduktion (und da schließe ich jetzt die paratextuelle Rahmung, etwa durch Retweets, ein) sichtbar, die es möglicherweise im Fall von in Buchform veröffentlichten Texten auch gibt, die aber dort nicht so auffallen, weil die Konventionen, Normen und Affordanzen andere sind? Als literarische ${ }^{*} r$ Autor ${ }^{*}$ in ein Buch zu veröffentlichen heißt ja, dass ein Name auf dem Cover steht und alle anderen bestenfalls in einer möglichen Danksagung erwähnt werden. Unter anderem deswegen ist eine Übertragung vom einen ins andere Medium auch so schwierig: Auf der einen Seite steht die enge Kopplung von Autorschaft und abgeschlossenem Text im Fall des Buches, auf der anderen Seite die Veröffentlichung von Text in einem Medium, in dem jeder Text innerhalb eines sichtbaren sozialen Geflechts und in enger Nachbarschaft zu Texten anderer Autor*innen erscheint.

Ja genau, und man könnte noch viele weitere Unterschiede und Ähnlichkeiten finden. Im Sonderforschungsbereich „Transformationen des Populären“"19 interessieren wir uns vor allem für die ,Popularisierung zweiter Ordnung‘ die dabei passiert: Die Social-Media-Posts machen sich nicht irgendwie selbst populär, sie schaffen dies vor allem auch, indem sie ihre eigene Popularität popularisieren. Mehr Follower führen zu mehr Followern, mehr Retweets zu mehr Retweets und dies wirft jeweils die Frage von Wertigkeit auf. Vorformen davon findet man vor allem ab etwa 1950 mit den Charts. Auch vorher gibt es natürlich Verkaufszahlen und ähnliches, die Wertigkeitsfrage war aber in der Regel schnell beantwortet: Was populär ist, ist nieder. Eine Art ,Umkehrung der Beweislast ${ }^{*}$ findet etwa nach dem zweiten Weltkrieg statt. ,High` und ,low‘ lösen sich nicht auf, aber deren Verhältnisse werden anders aushandelbar, unter anderem auch, weil Popularitätskennzahlen immer mehr immer schon bereits vorliegen. Das qualitative Argument ist gewissermaßen konstitutiv late to the party - was nicht heißt, dass es sich nicht durchsetzt, aber es steht unter anderer Begründungspflicht, die eben nicht immer geleistet werden kann.

Typisch für die Social-Media-Plattformen scheint mir nun, dass es keinen zeitlichen Unterschied mehr gibt - oder zumindest nur noch einen marginalen zwischen der Publikation eines Textes und dessen Popularisierung zweiter Ordnung. Hier wirkt sich zudem ein Effekt aus, der mich als Medienwissenschaftler

19 O.V.: „SFB 1472,Transformationen des Populären““. https://gepris.dfg.de/gepris/projekt/ 438577023, 2021 [zuletzt eingesehen am 7.6.2021]. 
sehr interessiert: Das Internet im Allgemeinen und die Social-Media-Plattformen im Besonderen sind skalenfreie Netze, das heißt, es gibt kaum eine Begrenzung der Verknüpfungshäufigkeit zwischen Knotenpunkten. Im Straßennetz kommen in der Regel bis zu vier, vielleicht auch sechs oder acht Linien an einem Knotenpunkt zusammen. In skalenfreien Netzen hingegen macht es keine größeren Probleme, zehn, zehntausend oder zehn Millionen Linien in einem Knoten zu versammeln. $^{20}$

Man hat dadurch eine Art Paradoxie der freien Wahl, durch die sich die Häufigkeitsverteilung in diesen skalenfreien Netzen erklären lässt. Es gibt immer - und diesmal meine ich tatsächlich immer - eine sogenannte Power-Law-Distribution aus einem Long-Tail, mit extrem vielen Knoten und sehr wenigen Verknüpfungen (zum Beispiel Twitter-Accounts mit wenigen Followern), einem Middle-Tail einiger Knoten mit mittelvielen und dann einem extrem steilen Short-Tail oder ,Head“ mit sehr wenigen Knotenpunkten und extrem vielen Verknüpfungen. In der Ökonomie des Netzes bildet das ,Winner-takes-all markets' aus (wenige Firmen beherrschen globale Märkte, aber viele kleine besetzen viele kleine Nischen). In der digitalen Kultur erzeugt dies extrem populäre Akteur^innen und gleichzeitig eine extrem große Menge an gewissermaßen leicht Populären, und dies hängt mit der Popularisierung zweiter Ordnung zusammen: Populäres wird noch populärer, auch weil seine Popularität reflexiv inszeniert wird und Nicht-Populäres findet seine Nische auch weil seine Nischigkeit inszeniert wird.

So groß also die Kontinuitäten langer Dauer sind, so spezifisch ist doch die Popularitätsdynamik skalenfreier Netze: Etablierte Praktiken, die auf der Plattform reproduziert werden, ändern sich. Die Wiederholung des Alten im neuen Milieu ist eine Transformation.

Da läge also eine eigentlich paradoxe Kopplung von Verlangsamung und Geschwindigkeit: Die ständige Wiedervorlage einzelner Tweets durch die Benachrichtigung über Likes und Retweets etc. steht der minimalen zeitlichen Distanz zwischen Publikation von Tweets und deren Popularisierung zweiter Ordnung gegenüber, die die Wiedervorlagen überhaupt erst erzeugt. Schnelligkeit übersetzt sich in Langsamkeit und Langsamkeit in Schnelligkeit, weil die Benachrichtigungen sowohl die „Dauer" des einzelnen Tweets anzeigen als auch die Schnelligkeit seiner Popularisierung.

Ich möchte in dieser Hinsicht noch einmal auf literarische Texte zurückkommen. Es gibt auf Twitter eine ganze Reihe von Literatur-Bots. Im Zentrum steht für diese Bots, die selten andere Tweets liken oder anderen User*innen folgen, nicht das Soziale am sozialen Netzwerk. Manche posten einfach vorhandenes Textmaterial wie die Joyce-Bots @finnegansreader oder @ulyssesreader, andere modifizieren ihr Ausgangsmaterial wie die Proust-Bots @BotRecherche oder @LongtempsP. Auch hier würde der Satz „Die Wiederholung des Alten im neuen Milieu ist eine Transformation" passen. Während die Joyce-Bots mit über 9.000 bzw. 15.000 Followers

20 Vgl. Barabási/Bonabeau: „Scale-Free Networks“. 
relativ beliebt sind, führen die Proust-Bots eine Nischenexistenz weit jenseits vierstelliger Follower-Zahlen. Besonders interessant sind die Tweets, gerade die der Proust-Bots, nicht, sie sind aus dem Kontext gerissen, oft nicht pointiert genug, teilweise ergeben sie keinen Sinn. Die, die ihnen folgen, stellen damit letztlich nur ihre Zugehörigkeit zu einer bestimmten high brow culture aus. Im Fall der Proust-Bots etwa: „Ich weiß, was eigentlich der erste Satz der Recherche ist, deswegen verstehe ich das Konzept des Bots." Bots wären also, in deinen Worten, eine „Inszenierung von Nischigkeit“, die gerade funktioniert, weil sie sich nicht an die Konventionen des Schreibens auf Twitter halten. Trotzdem werden sie letztlich in die Logik der Plattform eingespeist. Gibt es denn deiner Ansicht nach Strategien, die es schaffen diese Logik, die letztlich die „Winner-takes-all markets“ fundiert, zu unterlaufen? Könnte sie in der Überspitzung des „Macho-Unternehmersprechs“ der Start-Up-Bubble liegen? Oder hilft da einfach: abmelden?

Zur Geschwindigkeit: Ganz genau, wobei ich das Verhältnis weniger als paradox bezeichnen würde; vielmehr als skalierbar. Als User`in kann man ständig zwischen den Tempi wählen, im Kleinen versinken, während man das Große unbesehen vorbeirauschen lässt (und anders herum).

Die Joyce-Bots scheinen mir ebenfalls eher kultursoziologisch interessant als literaturwissenschaftlich. Mich erinnert das ein wenig an den Joyce-Tourismus in Dublin, wo die Leute in Sweny's Pharmacy pilgern, um Zitronenseife zu kaufen und dann in Davy Byrne's Pub weiterziehen, um wie Leopold Bloom ein Gorgonzola-Sandwich einzunehmen. Kommodifizierte Signale der Hochkultur, die möglicherweise ein Gefühl kulturellen Dabeiseins vermitteln, vielleicht auch tatsächlich bei der persönlichen Werkerschließung helfen, sich selbst aber dem Text gegenüber eher trivialisierend verhalten. Allerdings ist das aus meiner Sicht gar nicht mal so nischig, denn diese Hochkultursignale sind ja tatsächlich das, was große Teile des Kulturbetriebs am Laufen halten; ihn nicht nur finanzieren, sondern ihm auch seine gesellschaftliche Legitimität verleihen.

Dazu in aller Kürze eine Vignette jenseits von Twitter: Mein letzter Museumsbesuch vor der Corona-Pandemie war im Wallraf-Richartz in Köln, dort waren vor allem Arbeiten aus dem Umfeld Rembrandts ausgestellt, denen sich ansehen ließ, was an Rembrandts Formen und Verfahren damals in diesem Milieu zirkulierte, also was eben etablierte, mitspielfähige Praktik war, an der auch Rembrandt teilnahm..$^{21}$ Gleichzeitig wurde dann im Vergleich am Fall einiger weniger Rembrandts sichtbar, was den alten Meister dann doch von seinem Umfeld unterscheidet. Als ethnografisch Interessierter habe ich natürlich an einer Führung teilgenommen, und es war wie immer: Der allergrößte Teil des zahlenden

21 Wallraf-Richartz Museum \& Fondation Corboud: o.T. https://www.wallraf.museum/ausstel lungen/rueckblick/2020/2019-11-01-inside-rembrandt/information/, o.J. [zuletzt eingesehen am 7.6.2021]. 
Publikums interessiert sich nur für die Blockbuster, zählt durch, wie viele Rembrandts denn nun geboten werden, und ob das eigentlich genug ist. Diejenigen, die solche Ausstellungen konzipieren, wissen natürlich genau, dass es stets ein Drahtseilakt ist: Um eine interessante Ausstellung ermöglichen zu können, muss gleichzeitig die Blockbuster-Culture bedient werden. Mehr oder weniger heimlich wird dem Publikum dann aber das kunsthistorisch Interessante untergejubelt.

High culture als populärer Blockbuster ist also längst Teil der Kalkulation, die man oft produktiv zu wenden versucht. Für die bildende Kunst funktioniert das auch auf Twitter gut, wie etwa durch den Account @PP_Rubens, der täglich Bilder mal mehr, mal weniger kanonischer Kunst postet, wenn die*der Künstle$r^{\star}$ in Geburtstag hat und so eine große Vielfalt auch jenseits der Blockbuster erreicht. Der Account hat 30.000 Follower, darunter viele unserer Kolleg^innen ich bin auch dabei und nehme die Tweets oft zum Anlass, mir bestimmte Arbeiten und den*die Maler`in genauer anzusehen. Literatur hingegen hat es da auf Twitter ungleich schwerer als bildende Kunst, weil der Literaturblockbuster viel leichter zur Farce gerät. Bildende Kunst funktioniert viel eher auch als Schnipsel, große Teile unserer ästhetisch anspruchsvollen Bildkultur basieren schon immer auf (schlechten) Kopien, die zirkulieren - das ist ja auch Bruno Latours Kommentar zu Benjamins Aura-Aufsatz: Es ist die Vielzahl schlechter Kopien, die die Aura des Originals mithervorbringt. ${ }^{22}$ Der literarische Schnipsel allerdings funktioniert bestenfalls noch als Insider-Joke, mit dem man sich wechselseitig versichern kann, seinen Kafka gelesen zu haben - oder zumindest den ersten Satz aus Die Verwandlung (1915).

In mancher Hinsicht könnte man also sagen, dass gerade die textzentrierte Plattform Twitter geradezu unliterarisch ist, weil der Anschluss an die ästhetische Erfahrung größter Teile der Literatur - im Gegensatz zu anderen Kunstformen - ziemlich unwahrscheinlich ist. Gerade deshalb erscheint es mir interessant, wie mit diesem Problem umgegangen wird; ich würde sogar sagen, dass es für die Literaturinszenierung auf Twitter praxisleitend ist, selbst kaum literarisch sein zu können. Autor^innen wie Stanišić oder Gorman spielen daher ganz anders mit Twitter, als sie literarisch schreiben. Dennoch sind wir uns sicher einig, dass es auf der Plattform schon auch poetische Erfahrung gibt, und die verlangt auch eine ziemliche Kunstfertigkeit des Schreibens - und auch des Lesens. Die geschieht aber nicht, oder eher in Ausnahmefällen, durch Anschluss an das literarische Feld. Aus meiner Sicht funktioniert das viel mehr wie Rap, also

22 Vgl. Bruno Latour/Adam Lowe: „Das Wandern der Aura - oder wie man das Original durch seine Faksimiles erforscht“ [2008]. Übers. v. Gudrun Dauner/Johannes Paßmann. In: Tristan Thielmann/Erhard Schüttpelz (Hg.): Akteur-Medien-Theorie. Bielefeld 2013, S. 511-530. 
eine Kunstform, die gerade nicht versucht, mit etablierten Verfahren mitzuhalten und sich nicht mit deren Maßstäben messen lässt.

Deshalb haben sie auch keine größeren Probleme mit der Popularitätslogik der Plattform, sie müssen nicht nischig sein. Wer allerdings als Literatin gelten will, muss dann auf dem Papier etwas anderes liefern. Und das finde ich oft auffällig, wie schlecht manche brillante Twitterer werden, wenn es an den langen Text geht. Aber das ist eben auch ein anderes Spiel. Erstaunlich ist dann eher, dass es Multitalente wie Stanišić gibt, die beide Register bedienen können. Mit anderen Worten geht es aus meiner Sicht nicht darum, die Popularitätslogiken zu unterlaufen, sich zu wehren, das wären ja höchst heteronome Akte, und natürlich geht es ebenso wenig darum, sich dem Like-Counter an den Hals zu werfen. Zentral scheint mir eher die Indifferenz, oder genauer: die Inszenierung von Indifferenz. 